\title{
Особенности изменений активации
} тромбоцитов у больных хронической шизофренией в зависимости от степени тяжести позитивных и негативных симптомов при формировании ремиссии

Цель исследования: сравнить изменения степени тялсести позитивных, негативных и психопатологических симптомов PANSS при выходе больных шизофренией из приступа с формированием ремиссии и степени выраженности параметра, оценивающего степень активации тромбоцитов у этих больных. Методы: использовали психометрическую шкалу оценки тяжести синдромов РАNSS, а также метод определения активации тромбоцитов, основанный на подсчете числа клеток на выходе из колонки, содержсащей сефарозу CL-2B. Результаты: в процессе изменения степени тяжести болезни, оцениваемой по шкале РАNSS состояния хронических больных шизофренией при формировании ремиссии, наблюдается возрастание активации тромбоцитов, определенной на основе вышеуказанного количественного параметра. Сравнение изменений параметра оценки активации тромбоцитов с изменениями оценки степени тяжести болезни продемонстрировало сходные статистически неразличимые ( $p>0,05)$ изменения степени тяжести позитивного синдрома и количественного параметра, оценивающего активацию тромбоцитов, от 1-го к 3-му визиту при формировании ремиссии. Число тромбоцитов на выходе из колонки у больных шизофренией при 3-м визите было приблизительно в 2 раза меньше, чем при 1-м ( $p$ <0,002). Для позитивной субшкалы PANSS показано уменьшение в 1,6 раза степени тяжести синдрома ( $p<0,0002)$. Вместе с тем выявлено отличие параметра оценки активации тромбоцитов и тяжести негативного синдрома в 3-м визите (p <0,034). Заключение: показано сходное изменение степени тяжести позитивного синдрома и параметра оценки активации тромбоцитов от 1-го к 3-му визиту при формировании ремиссии. Выявлено также отличие параметра оценки активации тромбоцитов и тяжести негативного синдрома в 3-м визите.

Ключевые слова: тромбоцит, иизофрения, ремиссия.

(Для цитирования: Брусов О.С., Злобина Г.П. Особенности изменений активации тромбоцитов у больных хронической шизофренией в зависимости от степени тяжести позитивных и негативных симптомов при формировании ремиссии. Вестник РАMН. 2015; 70 (3): 355-359. Doi: 10.15690/vramn.v70i3.1333)

\section{O.S. Brusov, G.P. Zlobina}

Mental Health Research Centre, Moscow, Russian Federation

\section{Peculiarities of Platelet Activation Changes in Patients with Chronic Schizophrenia, Depending on the Severity of Positive and Negative Symptoms during the Remission Formation}

Objective: The aim of our study was to compare the changes in the severity of positive, negative and psychopathological PANSS symptoms which occur at an outcome of an attack in schizophrenic patients along with remission formation and the degree of platelet activation in these patients. Methods: Psychometric scale of the Positive and Negative Syndrome Scale (PANSS) and the method of estimation of platelet activation based on the calculation of cells number after elution from the column with sepharose $C L-2 B$ were used. Results: The changes in the severity of the disease were estimated using the PANSS scale of chronic schizophrenic patients in remission formation. An increase in platelet activation was determined on the basis of the above described quantitative parameter. Comparison of changes in platelet activation parameter with changes in the disease severity rating detected similar statistically indistinguishable $(p>0.05)$ changes in the severity of positive symptoms and quantitative variable of platelet activation between the $1^{\text {st }}$ and the $3^{\text {rd }}$ visits in the period of remission formation. Platelet number after elution from the column during the $3^{\text {rd }}$ visit was approximately two times less than during the $1^{\text {st }}$ visit $(p<0.002)$. PANSS positive subscale shows a decrease of the severity of the syndrome by 1.6 times $(p<0.0002)$. In addition the difference between the platelet activation parameter and severity of the negative syndrome was fixed during the $3^{\text {rd }}$ visit $(p<0.034)$. Conclusion: Similar changes in the severity of positive syndrome and platelet activation parameter were presented from $1^{\text {st }}$ to $3^{\text {rd }}$ visit in the remission formation. A distinction between the platelet activation parameter and severity of the negative syndrome was fixed during the $3^{\text {rd }}$ visit.

Key words: platelet, schizophrenia, remission.

(For citation: Brusov O.S., Zlobina G.P. Peculiarities of Changes of Platelet Activation in Patients with Chronic Schizophrenia, Depending on the Severity of Positive and Negative Symptoms during the Formation of Remission. Vestnik Rossiiskoi Akademii Meditsinskikh Nauk = Annals of the Russian Academy of Medical Sciences. 2015; 70 (3): 355-359. Doi: 10.15690/vramn.v70i3.1333) 


\section{Обоснование}

Шизофрения - сложное многомерное заболевание с неуточненной этиологией $[1,2]$. Изучение ремиссии при шизофрении важно для выяснения процессов, приводящих к развитию болезни по мере ее течения. Изучение ремиссии важно также для разработки стандартных критериев ремиссии, которые необходимы для создания четких представлений о стандартах качества ожидаемой помощи больному, для его ориентации на выздоровление. В настоящее время в качестве стандартных широко используют критерии ремиссии, разработанные международной группой экспертов [3]. Согласно этим критериям, ремиссия представляет собой улучшение состояния больного по основным признакам и симптомам (симптоматическая ремиссия) до уровня, на котором остаточные симптомы не достигают порога, определяюшего первичный диагноз шизофрении. Предложенное определение «симптоматическая ремиссия» имеет ряд ограничений. Оно отражает лишь степень выраженности симптомов при ремиссии, но не определяет прогноз болезни, не охватывает процесс выздоровления, который крайне важен для уточнения статуса больного [2, 4].

В настоящее время оценку клинического статуса больного проводят по следующим шкалам: Positive and Negative Syndrome Scale (PANSS), Brief Psychiatric Rating Scale (BPRS), Clinical Global Impression (CGI) и др. [5, 6]. При этом для оценки степени выраженности симптомов больного по шкале PANSS используют полуколичественную ординальную шкалу рангов (обычно от 1 до 7) [6]. Следует отметить, что такой подход, в отличие от количественных методов, менее точен и не исключает субъективности врача. В связи с этим для поиска объективных количественных методов оценки качества ремиссии целесообразно обратиться к изучению процессов восстановления организма больного на клеточном или субклеточном уровне.

К одному из выдающихся достижений в области современной биологии следует отнести значительное расширение знаний о функциях тромбоцитов, которые, например, у млекопитающих стали рассматривать не только как клетки, ответственные за гемостаз, но и как клетки, активно участвующие в различных иммунных реакциях организма. Подобие структурных и функциональных свойств белка-транспортера серотонина на мембранах тромбоцитов и синапсах центральной нервной системы позволяет использовать тромбоцит в качестве экстрацеребральной модели серотонинергического синапса центральной нервной системы [7, 8].

Исследование тромбоцитов у больных шизофренией указывает на множественные изменения их функций $[9,10]$.

В нашей предыдущей работе [11] при изучении тромбоцитов больных хронической шизофренией с приступообразным течением болезни показано последовательное возрастание активации тромбоцитов в процессе выхода из приступа и формирования ремиссии, сопровождающееся уменьшением степени выраженности большинства симптомов болезни и, как следствие, уменьшением позитивных и негативных симптомов шизофрении, оцениваемых по шкале PANSS. В связи с вышеизложенным представлялось целесообразным оценить, как связано последовательное уменьшение степени выраженности болезни с последовательным возрастанием активации тромбоцитов (с уменьшением числа тромбоцитов на выходе из колонки, содержащей сефарозу CL-2B) у больных при формировании ремиссии.
На первом этапе работы в этом направлении было необходимо провести сравнение степени выраженности изменений количественного параметра, оценивающего активацию тромбоцитов хронических больных шизофренией при гель-фильтрации через сефарозу CL-2B, и изменений в баллах клинических параметров 30 позитивных, негативных и психопатологических симптомов PANSS при выходе больных из приступа и формирования ремиссии.

Целью исследования было произвести сравнение изменений степени тяжести позитивных, негативных и психопатологических симптомов, оцениваемых по шкале PANSS, при выходе хронических больных шизофренией из приступа и формирования ремиссии с изменением активации тромбоцитов у этих больных.

\section{Методы}

\section{Дизайн исследования}

Проведено когортное проспективное исследование.

\section{Критерии соответствия}

Обследованы мужчины, больные шизофренией, с выраженными когнитивными нарушениями. Поступивших в стационар больных в течение 1-го мес лечили рисперидоном. Критерием включения в исследование являлся положительный ответ на лечение рисперидоном (начало исследования - 1-й визит). В момент включения в исследование всем больным был поставлен диагноз параноидной шизофрении с эпизодическим течением и нарастающим дефектом или эпизодическим течением со стабильным дефектом (код по МКБ-10 F 20.01 или F 20.02).

\section{Условия проведения}

Все пациенты были госпитализированы в психиатрическую больницу № 14 г. Москвы в связи с развившимся психотическим приступом.

Взятие крови осуществляли из локтевой вены утром, натощак, в пластмассовые флаконы, содержащие цитратный антикоагулянт $(1: 10)$. Обогащенную тромбоцитами плазму получали стандартным способом путем центрифугирования крови при $280 \mathrm{~g}$ в течение 15 мин при комнатной температуре. Суспензию тромбоцитов получали гель-фильтрацией с использованием сефарозы CL-2B [12]. Подсчет клеток проводили, как описано ранее [13], в камере Горяева на световом микроскопе фирмы Leitz (Германия) при 400-кратном увеличении. Подсчитывали не менее 150 клеток при каждом измерении.

Этапы исследования:

- изучение тромбоцитов у отобранных пациентов при лечении рисперидоном в качестве единственного антипсихотика в терапевтической схеме в течение 1-го мес (визит 1 - на высоте приступа);

- посещение врача через 1 мес, в течение которого проводили лечение рисперидоном (визит 2 - этап значительного улучшения состояния больного и начало формирования ремиссии);

- посещение врача через 3 мес лечения рисперидоном на этапе сформированной лекарственной ремиссии (визит 3).

\section{Продолжительность исследования}

Продолжительность исследования составила 3 мес. 


\section{Исходы исследования}

По мере формирования ремиссии у больных наблюдали заметное улучшение состояния, которое сопровождалось уменьшением степени выраженности симптомов, оцениваемых по шкале PANSS. Параллельно с оценкой степени тяжести болезни в 1, 2 и 3-м визитах определяли степень активации тромбоцитов, пропуская обогащенную тромбоцитами плазму (ОТП) через колонку, содержащую сефарозу CL-2B, и наблюдали последовательное возрастание активации тромбоцитов.

\section{Методы регистрации исходов}

Для оценки степени тяжести состояния больного использовали психометрическую шкалу оценки тяжести симптомов PANSS [6]. Шкала состоит из 3 подшкал, включающих по 7 симптомов для оценки позитивного и негативного синдромов и 16 симптомов для оценки общего психопатологического синдрома. Выраженность симптома оценивают по семибалльной системе: от 1 (отсутствие признака) до 7 (крайняя степень выраженности). Активацию тромбоцитов оценивали по числу клеток на выходе из колонки, содержащей сефарозу CL-2B.

Для сравнения изменений числа тромбоцитов на выходе из колонки и значений баллов, определяющих степень тяжести симптомов при формировании ремиссии, была использована следующая процедура. За $100 \%$ принимали как медианное число тромбоцитов, так и медианные значения баллов, определяющих степень выраженности симптома в 1-м визите. Значения на 2-м и 3-м визитах рассчитывали в процентах от значения, составляющего $100 \%$ (т.е. от медианных значений числа элюировавших тромбоцитов и балльных оценок симптомов при 1-м визите).

\section{Этическая экспертиза}

Разрешение на проведение эксперимента было представлено Этическим комитетом Научного центра психического здоровья (Москва), протокол № 89 от 28.03.2007 г.

\section{Статистический анализ}

Оценку результатов экспериментов осуществляли с использованием пакета программ STATISTICA v. 8.0 (StatSoft Inc., США). Распределение значений экспериментальных данных не являлось нормальным, в связи с этим для обсчета результатов применяли тесты непараметрической статистики. Данные отображены на рис., на котором представлены значения медианы $(\mathrm{Me})$ и значения переменной в 1-м и 3-м квартилях (Q1; Q3). В случае множественных сравнений при оценке различий числа тромбоцитов после выхода из колонки и значений степени тяжести симптомов при 1, 2 и 3-м визитах использовали дисперсионный анализ Фридмана с поправкой на множественные сравнения по Бонферрони. В случае оценки пар зависимых переменных применяли тест Вилкоксона. Различия считали статистически значимыми при $p<0,05$.

\section{Результаты}

\section{Объект исследования}

Исследованы тромбоциты 39 больных шизофренией мужчин с выраженными когнитивными нарушениями в

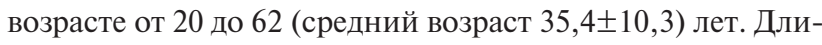
тельность болезни в среднем составила $9,0(5,0 ; 15,5)$ лет.

Bсе пациенты были госпитализированы в психиатрическую больницу в связи с развившимся психотическим приступом. В клинической картине у них имели место признаки галлюцинаторно-параноидного или параноидного синдрома.

До начала исследования с целью купирования приступа все больные получали антипсихотическую терапию, преимущественно типичными нейролептиками.

Исследование проводили с момента перевода на антипсихотическую монотерапию рисперидоном в терапевтических дозировках до формирования ремиссии. Дозы варьировали от 4 до $6(5,15 \pm 0,8)$ мг. Помимо рисперидона в терапевтическую схему могли быть включены другие

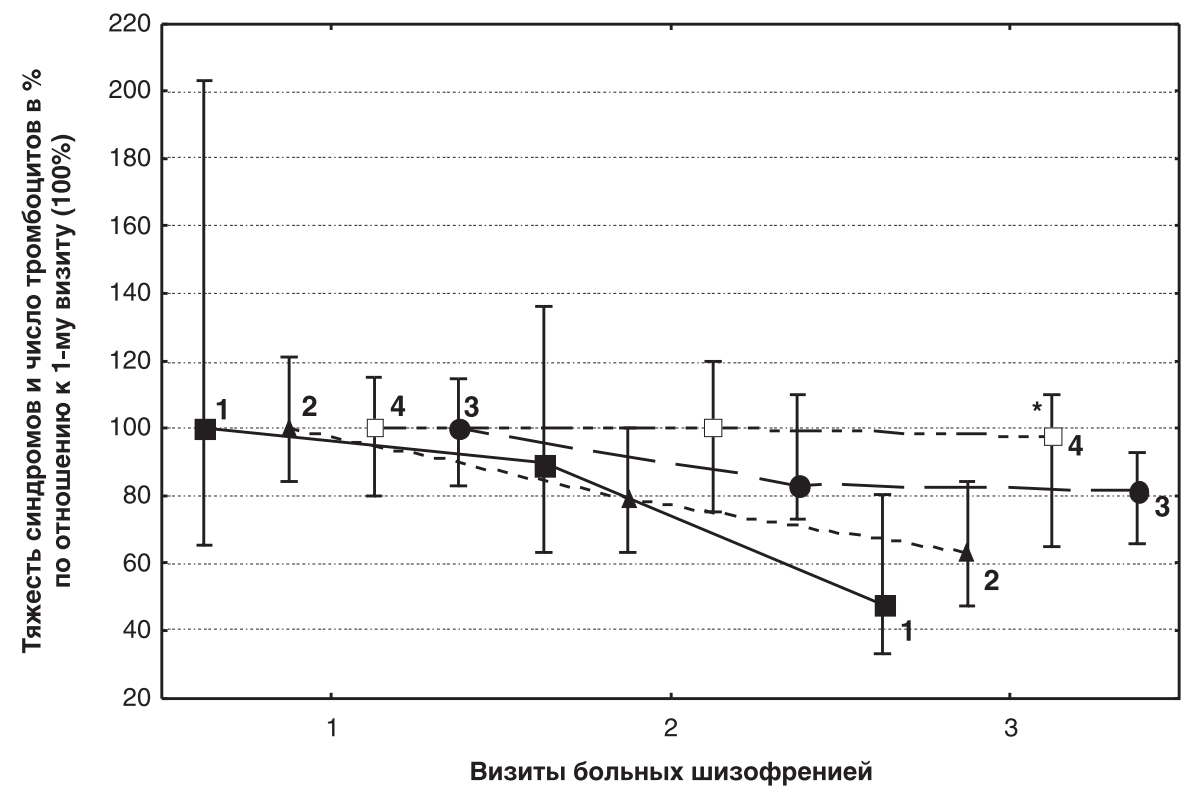

Рис. Изменение степени тяжести синдромов, оцениваемых по шкале PANSS, и число тромбоцитов после гель-фильтрации при формировании ремиссии у больных шизофренией.

Примечание. Кривая 1 - число тромбоцитов в процентах, элюировавших с колонки; кривая 2 - сумма баллов в процентах для позитивного синдрома; кривая 3 - сумма баллов в процентах для психопатологического синдрома; кривая 4 - сумма баллов в процентах для негативного синдрома. На рисунке представлены значения медианы. Вертикальные линии представляют значения переменной в 1-м и 3-м квартилях; * - достоверная $(p<0,034)$ разница по сравнению с числом тромбоцитов при 3-м визите. 
психотропные средства (например, антидепрессанты, транквилизаторы) в соответствии с требованиями актуального клинического состояния больных.

\section{Основные результаты исследования}

В предыдущей работе [11] при изучении тромбоцитов хронических больных шизофренией с приступообразным течением болезни активацию тромбоцитов оценивали по числу клеток на выходе из колонки. Наблюдали уменьшение числа тромбоцитов, прошедших через колонку, от 1 -го к 3 -му визиту ( $p<0,002)$. Число тромбоцитов на выходе из колонки у больных шизофренией при 3-м визите было приблизительно в 2 раза меньше, чем при 1-м. По тесту Вилкоксона для пар зависимых переменных число тромбоцитов на всех визитах достоверно различалось: для 1 -го и 2-го визита $-p<0,017$, для 1-го и 3-го $-p=0,002$, для 2-го и 3-го - $p=0,005$ (см. рис., кривая 1).

В настоящей работе для сравнения был проведен аналогичный анализ на достоверность изменений в числе баллов, оценивающих степень тяжести симптомов, при формировании ремиссии от 1-го к 3-му визиту для всех 30 симптомов, учитываемых в шкале PANSS, с использованием анализа Фридмана.

Для позитивной субшкалы PANSS было показано достоверное уменьшение $(p<0,034)$ суммы баллов по 6 симптомам: бред, расстройства мышления, галлюцинации, возбуждение, идеи величия, враждебность (исключение составил симптом «подозрительность»).

Для негативной субшкалы PANSS показано достоверное $(p<0,033)$ уменьшение суммы баллов лишь для симптома «трудность в общении». Для остальных 6 симптомов (притупленный аффект, эмоциональная отгороженность, апато-абулические расстройства, нарушения абстрактного мышления, нарушение спонтанности речи, стереотипное мышление) не показано достоверное уменьшение суммы баллов.

Для субшкалы общих психопатологических симптомов только 7 симптомов показали достоверное $(p<0,027)$ уменьшение суммы баллов (тревога, напряжение, манерность, нелепые мысли, фантастические идеи, снижение критики к болезни, поглощенность психическими переживаниями). Остальные 10 симптомов (поглощенность здоровьем, чувство вины, депрессия, двигательная заторможенность, отказ от сотрудничества, дезориентировка, недостаток внимания и концентрации, снижение воли и инициативы, импульсивность, активное избегание контактов) не показали достоверного уменьшения $(p>0,05)$ суммы баллов.

Таким образом, в нашем эксперименте более половины симптомов PANSS (17 из 30) не продемонстрировали достоверного изменения степени тяжести при выходе в симптоматическую ремиссию при лечении рисперидоном.

На следующем этапе работы симптомы, составляющие отдельный синдром, были суммированы и также проанализированы на предмет достоверности изменения суммы баллов по шкале PANSS от 1-го к 3-му визиту. Для позитивной субшкалы PANSS показано уменьшение в 1,6 раза степени тяжести синдрома $(p<0,0001)$. По тесту Вилкоксона для пар зависимых переменных значения в баллах, определяющие степень тяжести синдрома, при всех визитах достоверно различались: для 1-го и 2-го $p<0,0001$, для 1-го и 3-го - $p<0,0001$, для 2-го и 3-го $p<0,006$ (см. рис., кривая 2).

Менее выраженное уменьшение (в 1,2 раза) степени тяжести болезни при выходе в ремиссию показано для субшкалы общепсихопатологических симптомов
( $p=0,001)$. По тесту Вилкоксона для пар зависимых переменных значения в баллах, определяющие степень тяжести синдрома, достоверно различались как между $1-\mathrm{M}$ и 2-м $(p<0,017)$, так и между 1 -м и 3-м визитами $(p<0,0008)$. Однако между 2-м и 3-м визитами достоверного различия в степени тяжести синдрома зафиксировано не было (см. рис., кривая 3).

Для негативной субшкалы PANSS при выходе больных из приступа и формирования ремиссии значимых изменений степени тяжести болезни не выявлено (см. рис., кривая 4).

Далее мы оценивали достоверность различий по Фридману числа тромбоцитов, элюировавших с колонки, и степень тяжести синдромов - позитивного, негативного и синдрома «общая психопатология» - на 2-м и 3-м визитах. Для 2-го визита не показано достоверных различий между вышеупомянутыми переменными $(p>0,05)$. Анализ различий по Фридману числа тромбоцитов и значений в баллах степени тяжести 3 синдромов на 3-м визите показал их достоверное различие $(p<0,004)$. Использование критерия Вилкоксона продемонстрировало достоверное отличие числа тромбоцитов от степени тяжести негативного синдрома $(p<0,034)$ и не показало достоверного отличия от значений степени тяжести позитивного и общепсихопатологического синдрома в 3-м визите.

Таким образом, сравнение изменений количественного параметра с изменениями клинических параметров позволило установить его подобие с позитивным синдромом: значения переменных от 1-го к 3-му визиту не различались; для обеих переменных показаны достоверные отличия значений для 1-го и 2-го, 1-го и 3-го, 2-го и 3-го визитов.

\section{Обсуждение}

В настоящей работе проведено сравнение изменений клинических параметров, позволяющих соотнести степень тяжести позитивных, негативных и психопатологических симптомов по шкале PANSS, с изменениями количественного параметра, оценивающего степень активации тромбоцитов у хронических больных шизофренией при формировании ремиссии.

Анализ на достоверность различий в числе баллов, оценивающих степень тяжести болезни, от 1-го к 3-му визиту для 30 симптомов шкалы PANSS показал достоверное изменение степени тяжести лишь для 13 симптомов при выходе больных в симптоматическую ремиссию при лечении рисперидоном.

В нашей предыдущей работе [11] при изучении тромбоцитов хронических больных шизофренией с приступообразным течением болезни показано последовательное возрастание активации тромбоцитов в процессе выхода из приступа и формирования ремиссии. Активацию тромбоцитов оценивали при гель-фильтрации по числу клеток на выходе из колонки, содержащей сефарозу CL-2B: чем больше активация клеток, тем меньше число клеток, элюировавших с колонки, т.е. этот количественный метод может стать инструментом, позволяющим выявлять разную степень активации тромбоцитов в процессе формирования ремиссии и при дальнейшей разработке оказаться инновационным способом оценки качества ремиссии.

Сходные изменения степени тяжести позитивного синдрома и изменений количественного параметра, оценивающего активацию тромбоцитов от 1-го к 3-му визиту, и достоверное отличие параметра оценки активации тромбо- 
цитов от изменений степени тяжести негативного синдрома позволяют предположить участие тромбоцитов в механизмах формирования ремиссии у больных шизофренией.

Выход из психоза происходит с различной скоростью через разные домены, такие как симптомы, социальные отношения, профессиональное восстановление, качество жизни. При использовании стандартизованных критериев ремиссии показано [14-16], что они не отражают всех процессов, происходящих при ремиссии, в частности процесс выздоровления и прогноз болезни. В связи с вышеизложенным, ряд авторов [17-19] указывает на необходимость применения большего числа критериев, поскольку многие аспекты жизни больного изменяются при шизофрении, и использование ограниченного числа критериев приводит к неадекватному пониманию исхода болезни. Настоящая работа может быть отнесена к исследованиям, указывающим на возможность применения нового параметра для оценки качества ремиссии больных шизофренией. Новизна работы состоит в сравнении изменений количественного параметра (активации тромбоцитов), происходящих на клеточном уровне, с изменением клинической оценки степени тяжести болезни, оцениваемой по шкале PANSS при формировании ремиссии у больных шизофренией. Такой подход позволяет расширить и углубить наше понимание процессов, приводящих к формированию ремиссии у больных.

Возможной причиной появления в крови активированных тромбоцитов у больных шизофренией может быть изменение части клеток в результате выполнения ими защитных функций, которые сопровождаются их активацией или дегрануляцией. Обнаружение у больных шизофренией активации некоторых параметров врожденного иммунитета, выражающейся в увеличении активности эластазы в плазме крови, С-реактивного белка [9], позволяет предположить, что одной из причин активации тромбоцитов больных шизофренией является их участие в иммунных воспалительных реакциях.

\section{Заключение}

Разработка и применение биологических тестов, позволяющих давать точную количественную оценку статуса больного шизофренией в процессе развития болезни, представляет значительный интерес для установления стандартных критериев, характеризующих ремиссию. При проведении дополнительных исследований полученные результаты могут быть впоследствии использованы как новые параметры оценки качества ремиссии заболевания.

\section{Конфликт интересов}

Авторы данной статьи подтвердили отсутствие финансовой поддержки / конфликта интересов, о которых необходимо сообщить.

\section{ЛИТЕРАТУРА}

1. Davidson L., Schmutte T., Dinzeo T., Andres-Hyman R. Schizophr.Bull. 2008; 34 (1): 5-8.

2. Harvey C.A., Jeffreys S.E., McNaught A.S., Bizard R.A., King M.B. Int. J. Soc. Psychiatry. 2007; 53 (4): 340-356.

3. Andreasen N.C., Carpenter W.T., Kane J.M., Lasser R.A., Weinberger D.R. Am. J. Psychiatry. 2005; 162 (3): 441-449.

4. Emsley R., Chiliza B., Asmal L., Lehloenya K. Curr. Opin. Psychiatry. 2011; 24 (2): 114-121.

5. Mortimer A.M. Brit. J. Psychiatry. 2007; 191 (50): 7-14.

6. Kay S.R., Fiszbein A., Lindenmayer J.P., Opler L.A Acta Psychiatr. Scand. 1986; 74 (5): 507-518.

7. Habets K.L., Huizinga T.W., Toes R.E. Eur. J. Clin. Invest. 2013; 43 (7): 746-757.

8. Klinger M.H., Jelkmann W.J. Interferon. Cytokine Res. 2002; 22 (9): 913-922.

9. Щербакова И.В., Каледа В.Г., Бархатова А.Н., Клюшник Т.П. Журнал неврологии и психиатрии. 2005; 105 (3): $43-46$.

10. Бонарцев П.Д., Рахманова В.И., Безруков М.В., Фактор М.И., Брусов О.С., Уранова Н.А. Журнал неврологии и психиатрии. 2014; 114 (2): 42-46.
11. Брусов О.С., Злобина Г.П. Вестник РАМН. 2013; 9: $42-45$.

12. Киктенко А.И., Злобина Г.П., Щурин М.Р., Клещинов В.Н. Бюллетень экспериментальной биологии и медицины. 1991; 112 (11): 485-488.

13. Злобина Г.П., Брусов О.С., Морозова М.А., Бениашвили А.Г. Журнал неврологии и психиатрии. 2009; 109 (10): $47-50$.

14. Lambert M., Karow A., Leucht S., Schimmelmann B.G., Naber D. Dialogues Clin. Neurosci. 2010; 12 (3): 393-407.

15. Helldin L., Kane J.M., Karilampi U., Norlander T., Archer T. J. Psychiatr. Res. 2006; 40 (8): 738-745.

16. Wobrock T., Kohler J., Klein P., Falkai P. Acta Psychiatr. Scand. 2009; 120 (2): 120-128

17. Lehman A.F., Carpenter W.T., Goldman H.H., Steinwachs D.M. Schizophr. Bull. 1995; 21: 669-675.

18. Мосолов С.Н.,Потапов А.В., Дедюрина Ю.М., Ушаков Ю.В., Цукарзи Э.Э. Журнал неврологии и психиатрии. 2010; 110 (5): 71-75.

19. Gorwood P., Peuskens J. Eur. Psychiatry. 2012; 27 (3): $170-1$.

\section{КОНТАКТНАЯ ИНФОРМАЦИЯ}

Брусов Олег Сергеевич, кандидат биологических наук, заведующий лабораторией биохимии НЦ психического здоровья

Адрес: 117152, Москва, Загородное ш., д. 2, корп. 3, стр. 16, тел.: +7 (495) 952-91-41, e-mail: oleg_brusov@mail.ru Злобина Галина Петровна, кандидат биологических наук, старший научный сотрудник лаборатории биохимии НЦ психического здоровья

Адрес: 117152, Москва, Загородное ш., д. 2, корп. 3, стр. 16, тел.: +7 (495) 952-91-41, e-mail: zlobina2000@mail.ru 